\title{
Expression pattern of the SARS-CoV-2 receptor ACE2 and TMPRSS2 in the respiratory tract
}

Yichuan Liu ${ }^{1 *}$, Hui-Qi Qu ${ }^{1 *}$, Lifeng Tian ${ }^{1}$, Hakon Hakonarson ${ }^{1,2 \dagger}$

${ }^{1}$ Center for Applied Genomics, The Children's Hospital of Philadelphia, Philadelphia, Pennsylvania, USA

${ }^{2}$ Divisions of Human Genetics and Pulmonary Medicine, Department of Pediatrics, The Perelman School of Medicine, University of Pennsylvania, Philadelphia, Pennsylvania, USA

* Both authors contributed equally to this work.

$\dagger$ Corresponding authors:

Dr. Hakon Hakonarson

Center for Applied Genomics, The Children's Hospital of Philadelphia, Philadelphia,

Pennsylvania, United States of America, Department of Pediatrics, University of Pennsylvania, Philadelphia, Pennsylvania, United States of America

Email: $\underline{\text { Hakonarson@chop.edu }}$

\begin{abstract}
To address the expression pattern of the SARS-CoV-2 receptor ACE2 and the viral priming protease, TMPRSS2, in the respiratory tract, this study investigated RNA sequencing transcriptome profiling of samples of airway and oral mucosa. As shown, ACE2 has medium levels of expression in both small airway epithelium and masticatory mucosa, and high levels of expression in nasal epithelium. The expression of ACE2 is low in mucosal associated invariant T (MAIT) cells, and can't be detected in alveolar macrophages. TMPRSS2 is highly expressed in small airway epithelium and nasal epithelium, and has lower expression in masticatory mucosa. Our results highlights that the nasal mucosa is the most susceptible locus in the respiratory tract for SARS-CoV-2 infection and consequently for subsequent droplet transmission and should be the focus for protection against SARS-CoV-2 infection.
\end{abstract}

Key words: ACE2; COVID-19; SARS-CoV-2; TMPRSS2 


\section{Introduction}

Concerning the pandemic of Coronavirus Disease 2019 (COVID-19), on April $23^{\text {rd }}, 2020$, it has been diagnosed in $>2.7$ million people globally, causing $>189,000$ deaths. COVID-19 is caused by the infection of the severe acute respiratory syndrome coronavirus 2 (SARS-CoV-2). For this highly infectious and deadly disease, there is no effective anti-viral treatment, and the mortality rate is high, or 2-3\%[1]. The human angiotensin I converting enzyme 2 (ACE2) has been suggested to serve as the receptor for the cell entry of SARS-CoV-2 to cause infection[2]. The ACE2 gene maps to ChrX. ACE2 is a member of the renin-angiotensin system (RAS), with the function of converting angiotensin II to angiotensin-(1-7) (with 7 amino acids), and converting angiotensin I to angiotensin-(1-9)[3], thereby negatively regulating the effects of angiotensin I converting enzyme (ACE) and the RAS system. In addition to its critical roles in RAS, ACE2 binds the S1 domain of the SARS-CoV Spike (S) protein as the viral receptor, and accounts for the infection of SARS-CoV and syncytia formation[4]. The genome sequence of SARS-CoV-2 shows significant similarity (79\%) to that of SARS-CoV, while its receptorbinding domain shows even higher similarity to that of SARS-CoV[2], further supporting ACE2 as the receptor of SARS-CoV-2. COVID-19 is a highly infectious respiratory disease with a basic reproduction number R0 $(95 \% \mathrm{CI})$ of $3.28(1.4,6.49)[5]$. After binding with ACE2, SARS$\mathrm{CoV}-2$ priming by the serine protease encoded by the transmembrane serine protease 2 gene (TMPRSS2) is also required for the viral entry into host cells[6, 7]. Knowledge about the expression of $A C E 2$ and TMPRSS2 is extremely important to understand the infection of SARS$\mathrm{CoV}-2$, and to find ways to prevent the infection. For this purpose, we investigated RNA sequencing transcriptome profiling of samples of airway and oral mucosa, including small airway epithelium, alveolar macrophages, nasal epithelium, and masticatory mucosa. In addition, considering the critical role of mucosal associated invariant T (MAIT) cells in mucosal immune defense against viral infection[8], transcriptome profiling of MAIT was also examined in this study.

\section{Methods}

Five datasets of transcriptome profiling by RNA sequencing (RNAseq) were acquired from the NCBI Gene Expression Omnibus (GEO) database (Table 1). We mapped and quantified the trimmed RNA-SEQ reads using HISAT2 (https://ccb.jhu.edu/software/hisat2/index.shtml) to hg19 refSeq for each sample at default thresholds. The expression matrix was generated based on Cuffnorm functions in Cufflink package version 2.2.1[9]. Library sizes (i.e. sequencing depths) are normalized by the classic-fpkm method. Comparisons of the levels of ACE, ACE2, and TMPRSS2, across different samples were based on the control or pre-exposure samples in each dataset, i.e. small airway epithelium of 10 healthy never-smokers before smoking E-cigarette; alveolar macrophages of 10 healthy never-smokers before smoking E-cigarette; nasal epithelium of 4 non-smoker females before exposure to third hand smoke; masticatory mucosa of 21 never smokers; and MAIT of 5 healthy bodyweight donors. The relative levels of the two genes were presented as the Fragments Per Kilobase of transcript per Million mapped reads (FPKM); all values were corrected by the average of relative levels of 6 house-keeping genes, i.e. $A C T B$, GAPDH, HMBS, HPRT1, RPL13A, and TBP.

\section{Results and Discussion}


Gene expression patterns in five types of normal tissues are shown in Fig.1. ACE and ACE2 have medium and comparable levels of expression in both small airway epithelium and masticatory mucosa. These findings suggest that SARS-CoV-2 can infect both small airway epithelium and oral mucosa. The ACE2 expression with the highest level of TMPRSS2 expression in small airway epithelium provides explanation for the vulnerability infected individuals have for the characteristic pneumonia of COVID-19. The ACE2 expression in masticatory mucosa helps explain the high level of infectivity via droplet transmission from SARS-CoV-2 infection residing in the oral mucous membrane. Interestingly, the expression level of both ACE2 and TMPRSS2 in nasal epithelium is much higher than the levels of $A C E$ expression. These results provide mechanistic evidence that the SARS-CoV-2 virus resides in both the oral and nasal mucosa of the upper respiratory tract where it is able to bind to the ACE2 receptor and serving as the principal locus of infections. These results also provide explanation for the high level of viral load in the oral and nasal mucosa and resulting high level of droplets transmission, with the lower airways being responsible for the severe form of pneumonia as well as the aerosol transmission of the virus, which may have implications for the prevention measures of SARS-CoV-2 viral transmission.

The expressions of ACE, ACE2, and TMPRSS2 are low in MAIT cells. The expression of $A C E$ is high in alveolar macrophages, but the expression of $A C E 2$ can't be detected with a low level of TMPRSS 2 in alveolar macrophages. These patterns of gene expression in the two types of innate immune cells suggest that SARS-CoV-2 has no or little direct impact on these two components of the innate immune system. In addition, these findings suggest that the infection of SARS-CoV-2 can be limited to the respiratory tract, which explains the absent of viremia in many COVID-19 patients[10, 11].

In parallel, we examined whether the exposure factor in each RNAseq dataset affected the expression levels of ACE, ACE2, and TMPRSS2, to assess whether those common factors (i.e. smoking E-cigarette, third hand smoke, smoking, and obesity) are associated with the susceptibility of SARS-CoV-2 infection. FPKM values of ACE, ACE2, and TMPRSS2, within each dataset were compared by paired T test (for GSE85121 and GSE129959) or independent T test (for GSE136262 and GSE126169). However, there was no difference observed $(P>0.05)$ between the dataset for ACE, ACE2, or TMPRSS2. Accordingly, while the SARS-CoV-2 virus is highly infectious, our results do not suggest significant change of infectious susceptibility of SARS-CoV-2 infection by these factors. However, because of the modest sample size for each datasets, we must acknowledge that we are underpowered to identify minor effects.

In summary, this study highlights that the nasal mucosa is the most susceptible locus in the respiratory tract for SARS-CoV-2 infection and replication, responsible for the subsequent high level of droplet transmission and should be the focus for protection against SARS-CoV-2 infection, in line with a recently virological analysis[12]. Accordingly, local interventions with ACE2 inhibitor[13] or TMPRSS2 inhibitor (e.g. camostat mesylate)[7] may represent novel interventions to block SARS-CoV-2 cell entry and treat COVID-19.

\section{Declarations}

Ethics approval and consent to participate 
Not applicable

\section{Consent for publication}

Not applicable

\section{Availability of data and materials}

All data generated or analysed during this study are included in this published article.

\section{Competing interests}

The authors declare that they have no competing interests

\section{Funding}

This study was funded by an Institute Development Fund to the Center for Applied Genomics (CAG) at The Children's Hospital of Philadelphia.

\section{Authors' contributions}

YL, HQ analyzed and interpreted the RNAseq data. YL, HQ, LT, and HH wrote the manuscript. All authors read and approved the final manuscript.

\section{Acknowledgements}

Not applicable 
Table 1 Five datasets of RNAseq profiling analyzed in this study

\begin{tabular}{|l|l|l|}
\hline $\begin{array}{l}\text { GEO } \\
\text { accession }\end{array}$ & Sample & Data description \\
\hline GSE85121 & $\begin{array}{l}\text { small airway } \\
\text { epithelium[14] }\end{array}$ & $\begin{array}{l}\text { 10 healthy never-smokers before and after smoking E- } \\
\text { cigarette }\end{array}$ \\
\hline GSE85121 & $\begin{array}{l}\text { alveolar } \\
\text { macrophages[14] }\end{array}$ & $\begin{array}{l}10 \text { healthy never-smokers before and after smoking E- } \\
\text { cigarette }\end{array}$ \\
\hline GSE129959 & nasal epithelium & $\begin{array}{l}\text { 4 non-smoker females before and after exposure to } \\
\text { thirdhand smoke }\end{array}$ \\
\hline GSE136262 & $\begin{array}{l}\text { masticatory } \\
\text { mucosa[15] }\end{array}$ & 21 never smokers; 17 current smokers \\
\hline GSE126169 & MAIT[8] & 5 healthy bodyweight donors; 4 morbidly obese donors \\
\hline
\end{tabular}


60

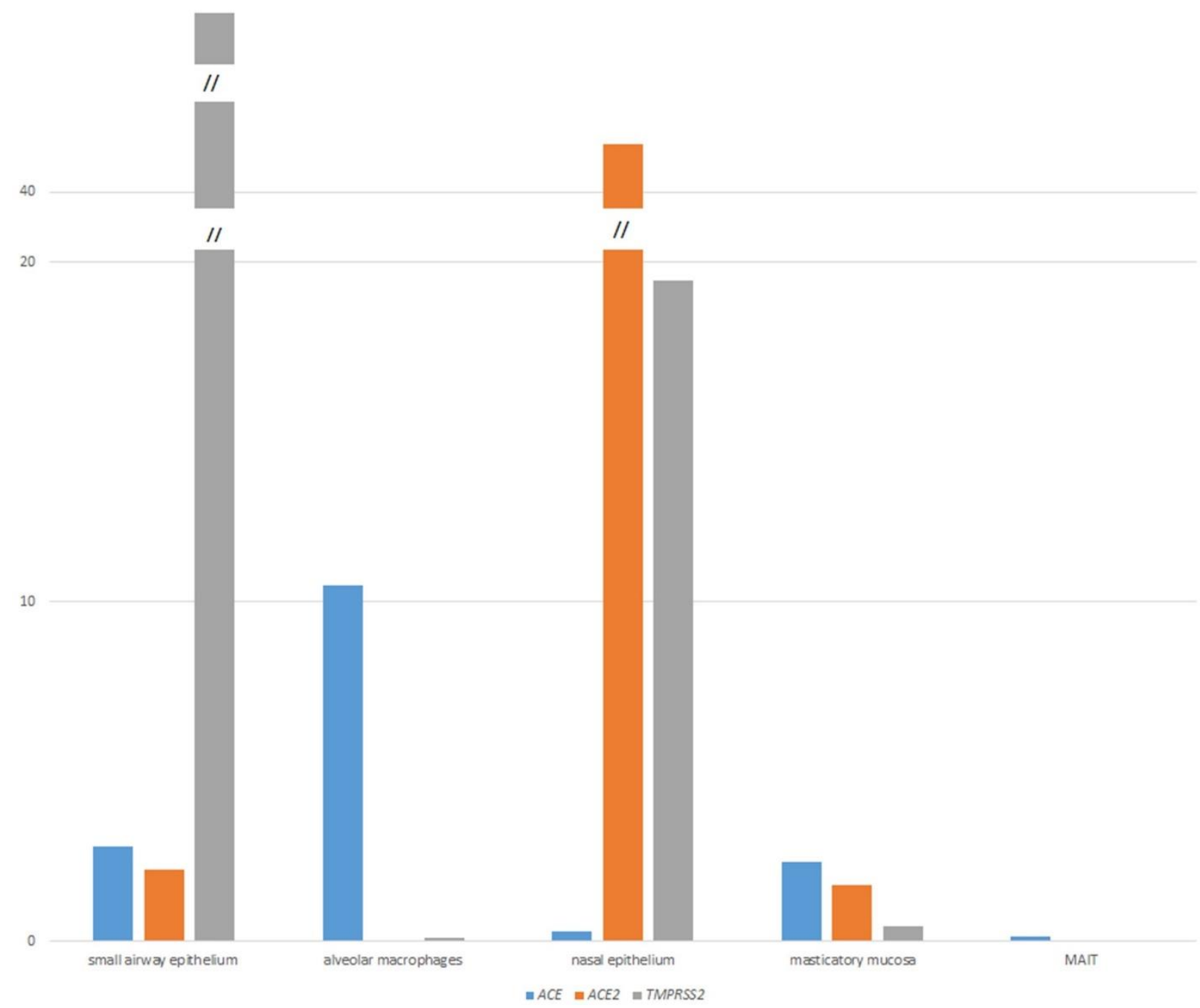

Figure 1. The expression of $A C E, A C E 2$, and TMPRSS2 in five different types of samples. $\mathrm{Y}$-axis represents FPKM values. 
References:

1. Wu Z, McGoogan JM: Characteristics of and Important Lessons From the Coronavirus Disease 2019 (COVID-19) Outbreak in China: Summary of a Report of 72314 Cases From the Chinese Center for Disease Control and Prevention. JAMA 2020.

2. L Lu R, Zhao X, Li J, Niu P, Yang B, Wu H, Wang W, Song H, Huang B, Zhu N: Genomic characterisation and epidemiology of 2019 novel coronavirus: implications for virus origins and receptor binding. The Lancet 2020.

3. Ferrario CM, Ahmad S, Nagata S, Simington SW, Varagic J, Kon N, Dell'italia L: An evolving story of angiotensin-II-forming pathways in rodents and humans. Clinical science (London, England: 1979) 2014, 126:461-469.

4. Li W, Moore MJ, Vasilieva N, Sui J, Wong SK, Berne MA, Somasundaran M, Sullivan JL, Luzuriaga $\mathrm{K}$, Greenough TC: Angiotensin-converting enzyme 2 is a functional receptor for the SARS coronavirus. Nature 2003, 426:450-454.

5. Liu Y, Gayle AA, Wilder-Smith A, Rocklöv J: The reproductive number of COVID-19 is higher compared to SARS coronavirus. Journal of travel medicine 2020.

6. Lukassen S, Chua RL, Trefzer T, Kahn NC, Schneider MA, Muley T, Winter H, Meister M, Veith C, Boots AW: SARS-CoV-2 receptor ACE2 and TMPRSS2 are primarily expressed in bronchial transient secretory cells. The EMBO Journal 2020.

7. Hoffmann $M$, Kleine-Weber $H$, Schroeder S, Krüger N, Herrler T, Erichsen S, Schiergens TS, Herrler $\mathrm{G}, \mathrm{Wu} \mathrm{N}-\mathrm{H}$, Nitsche A: SARS-CoV-2 cell entry depends on ACE2 and TMPRSS2 and is blocked by a clinically proven protease inhibitor. Cell 2020.

8. O'Brien A, Loftus RM, Pisarska MM, Tobin LM, Bergin R, Wood NAW, Foley C, Mat A, Tinley FC, Bannan C, et al: Obesity Reduces mTORC1 Activity in Mucosal-Associated Invariant T Cells, Driving Defective Metabolic and Functional Responses. J Immunol 2019, 202:3404-3411.

9. Trapnell C, Williams BA, Pertea G, Mortazavi A, Kwan G, van Baren MJ, Salzberg SL, Wold BJ, Pachter $L$ : Transcript assembly and quantification by RNA-Seq reveals unannotated transcripts and isoform switching during cell differentiation. Nat Biotechnol 2010, 28:511-515.

10. Lescure F-X, Bouadma L, Nguyen D, Parisey M, Wicky P-H, Behillil S, Gaymard A, BouscambertDuchamp M, Donati F, Le Hingrat Q: Clinical and virological data of the first cases of COVID-19 in Europe: a case series. The Lancet Infectious Diseases 2020.

11. Chang L, Yan Y, Wang L: Coronavirus Disease 2019: Coronaviruses and Blood Safety. Transfusion Medicine Reviews 2020.

12. Ehmann KZ, Drosten C, Wendtner C, Zange M, Vollmar P, Rosina Ehmann D, Zwirglmaier K, Guggemos M, Seilmaier M, Niemeyer D: Virological assessment of hospitalized cases of coronavirus disease 2019.

13. Huentelman MJ, Zubcevic J, Hernandez Prada JA, Xiao X, Dimitrov DS, Raizada MK, Ostrov DA: Structure-based discovery of a novel angiotensin-converting enzyme $\mathbf{2}$ inhibitor. Hypertension 2004, 44:903-906.

14. Staudt MR, Salit J, Kaner RJ, Hollmann C, Crystal RG: Altered lung biology of healthy never smokers following acute inhalation of E-cigarettes. Respir Res 2018, 19:78.

15. Richter GM, Kruppa J, Munz M, Wiehe R, Hasler R, Franke A, Martins O, Jockel-Schneider Y, Bruckmann C, Dommisch H, Schaefer AS: A combined epigenome- and transcriptome-wide association study of the oral masticatory mucosa assigns CYP1B1 a central role for epithelial health in smokers. Clin Epigenetics 2019, 11:105. 